Arq. Bras. Med. Vet. Zootec., v.70, n.3, p.749-757, 2018

\title{
Angiografia dos dígitos de bovinos jovens antes e após indução de acidose ruminal
}

[Digital angiography in young cattle before and after ruminal acidosis induction]

S.L.R. Freitas, N.C. Borges, A.D.F. Noronha Filho, J.R. Cardoso, P.J.B. Queiroz, R.E. Rabelo, L.A.F. Silva

Universidade Federal de Goiás - Goiânia, GO

\section{RESUMO}

O presente estudo objetivou descrever a rede vascular dos dígitos dos membros pélvicos de bovinos antes e após aplicação intrarruminal de oligofrutose, empregando a venografia. Utilizaram-se seis bovinos alocados em dois grupos (GI e GII), que receberam 13 e $17 \mathrm{~g} / \mathrm{kg}$ de oligofrutose, respectivamente. A venografia dos dígitos foi feita em quatro momentos: 15 dias antes (M0) e 36 horas (M1), sete dias (M2) e 30 dias (M3) após aplicação de oligofrutose. Mensurou-se a largura das veias visualizadas e avaliou-se possível supressão do fluxo sanguíneo dos vasos. A largura foi avaliada pela análise de variância (ANOVA) e pelo teste $\mathrm{t}(5 \%)$. Em M0, nas radiografias, foram identificadas 25 estruturas, incluindo veias, artérias e rede vascular. As veias mensuradas foram: digital dorsal comum III e dorsal da falange distal e os ramos plantares para o coxim digital do dígito lateral e medial. Apenas a v. digital dorsal comum III apresentou maior largura após 36 horas e constrição com 30 dias $(\mathrm{P} \leq 0,05)$ da aplicação de oligofrutose. Comparando-se os momentos, encontrou-se vasodilatação no M1 e vasoconstrição nos demais. A mensuração da largura dos vasos não sofreu $(\mathrm{P} \geq 0,05)$ interferência quando se compararam os grupos. Concluiu-se que a venografia dos dígitos de bovinos permite estudar e identificar alterações da vascularização.

Palavras-chave: anatomia, bovino, laminite, oligofrutose, radiografia

\begin{abstract}
The objective of this study was to describe vascular net of bovine digit before and after intrarruminal administration of oligofructose, with the use of venography. Six animals sorted in two groups (GI and GII) that received 13 and $17 \mathrm{~g} / \mathrm{kg}$ of oligofructose respectively were used. Digits venography were done in four moments: 15 days before (M0) and 36 hours (M1), seven days (M2) and 30 days (M3) after administration of oligofructose. Width of visualized veins and evaluated possible blood flow vessels suppression was measured. The width was evaluated by ANOVA and T test (5\%). In MO 25 structures were identified, including veins, arteries and vascular net in radiographic images. Veins measured were: common digital III and dorsal of distal phalanx and plantar branches to digital cushion of lateral and medial digits. Only V. digital dorsal common III presented higher width after 36 hours and constriction with 30 days $(P \leq 0.05)$ of oligofructose administration. Comparing moments, vessel dilation on M1 and constriction on other moments. Measurement of vessel width didn't suffer interference when groups were compared. Venography of bovine digits allowed for the study and identification of changes in vascularization.
\end{abstract}

Keywords: anatomy, bovine, laminitis, oligofrutctose, radiography

\section{INTRODUÇÃO}

A laminite é uma doença multifatorial e alguns fatores de risco envolvidos na gênese do problema ainda são motivos de especulações

Recebido em 7 de fevereiro de 2017

Aceito em 25 de agosto de 2017

E-mail: sabrina.freitas@ifgoiano.edu.br
(Danscher et al., 2009). No estudo da laminite, a oligofrutose, carboidrato de alta fermentação, tem sido uma alternativa viável na indução do problema. A acidose ruminal desencadeada por esse carboidrato permite estudar alterações sequenciadas que ocorrem durante a instalação 
da doença (Thoefner et al., 2004, 2005; Danscher et al., 2009). Nos dígitos, podem-se destacar as alterações vasculares comprovadas pela arteriografia em peças obtidas em matadouros.

O exame revelou modificações vasculares importantes nos dígitos de bovinos com laminite (Nurbakhsh et al., 2009). Igualmente, a venografia realizada em animais vivos tem revelado modificações relevantes que ocorrem nos vasos sanguíneos do dígito de equinos portadores da afecção (Redden, 2001a; Baldwin e Pollitt, 2010).

Porém, informações existentes envolvendo a anatomia vascular dos dígitos de bovinos, saudáveis e portadores de laminite, e as alterações que ocorrem desde a instalação da doença até a fase crônica são insuficientes para a compreensão de todos os mecanismos envolvidos na gênese do processo. Dessa forma, o presente estudo objetivou empregar a venografia nos dígitos de bovinos, antes e após a administração intrarruminal de oligofrutose, com a finalidade de delimitar a anatomia vascular hígida e, sequencialmente, avaliar possíveis alterações vasculares dos dígitos desses animais.

\section{MATERIAL E MÉTODOS}

O estudo foi desenvolvido no Setor de Grandes Animais do Hospital Veterinário da Escola de Veterinária e Zootecnia da Universidade Federal de Goiás (SGA/HV/EVZ/UFG), após o projeto ter sido aprovado pelo Comitê em Ética no Uso de Animais (Ceua), processo número 26/2013. Foram empregados na pesquisa seis animais mestiços (Bos indicus x Bos taurus), machos, inteiros, com 12,6 $6 \pm 0,9$ meses de idade e peso de $156 \pm 37,8 \mathrm{~kg}$, manejados em piquetes com gramínea Brachiaria decumbens e água à vontade. Antes do início da pesquisa, os animais foram submetidos à ruminostomia para colocação de cânulas. Após cicatrização clínica da ferida cirúrgica, foram distribuídos em dois grupos (GI e GII) de três animais, de acordo com a dose de oligofrutose (Oligofrutose $\AA$, Viafarma, São Paulo, Brasil) recebida via intrarruminal. As doses foram de $13 \mathrm{~g} / \mathrm{kg}$ para os animais do GI e de $17 \mathrm{~g} / \mathrm{kg}$ para os do GII (Thoefner et al., 2004).

Foram realizadas avaliações do $\mathrm{pH}$ ruminal e hemogasometria para comprovação da acidose ruminal. $\mathrm{O}$ exame foi realizado antes e a cada seis horas após oferecimento da oligofrutose, até serem completadas 36 horas. Foi feito também teste de claudicação, adaptado de Sprecher et al. (1997), antes e a cada 12 horas, por 72 horas após aplicação intrarruminal de oligofrutose. A venografia dos dígitos dos bovinos foi realizada antes e após a aplicação da oligofrutose, sempre no período da manhã, empregando-se apenas o membro pélvico esquerdo. A avaliação ocorreu em quatro momentos distintos, sendo 15 dias antes (M0) da aplicação da oligofrutose e após 36 horas (M1), sete dias (M2) e 30 dias (M3) da administração do carboidrato. $\mathrm{O}$ momento $\mathrm{M} 0$ de cada animal serviu como controle para as avaliações subsequentes.

Para a venografia, os animais foram sedados com cloridrato de xilazina $2 \%$ (Calmiun $\AA$, Agener União - Saúde Animal, São Paulo, Brasil) e contidos em decúbito lateral direito, com os membros imobilizados por cordas. O membro pélvico esquerdo foi imobilizado manualmente. Em seguida, realizou-se antissepsia para a canulação da v. digital dorsal comum III, com scalp $\mathrm{n}^{\circ}$ 21. O acesso à veia foi realizado na região dorsal da articulação metatarsofalangeana, após se aplicar um garrote de borracha no terço final da região metatársica. O scalp foi fixado com esparadrapo para manter a via de acesso, e aplicaram-se aproximadamente $2 \mathrm{~mL}$ de solução heparinizada $1 \%$ para evitar a formação de coágulos. Aspirava-se a quantidade de sangue que era possível e aplicavam-se $20 \mathrm{~mL}$ de contraste contendo $300 \mathrm{mg}$ de solução iodada (Iopamiron $\AA$, Iodopamidol, Bayer, Rio de Janeiro, RJ) de forma lenta. Uma pinça hemostática foi empregada para prender o scalp e evitar o refluxo de sangue ou do próprio contraste. Ato continuo, duas radiografias foram feitas, sendo uma em projeção dorsoplantar e, logo em seguida, outra em posição lateromedial. Toda a metodologia da realização da radiografia foi adaptada de Redden (2001b).

Os exames foram realizados com aparelho radiográfico portátil (Orange 1060HF VET $\AA$, Ecoray, Seoul, Coreia do Sul, programado para $125 \mathrm{kVp}$ e $25 \mathrm{mAs}$ ) calibrado em $48 \mathrm{kVp}, 2.5 \mathrm{mAs}$ e distância foco-filme de $70 \mathrm{~cm}$. Finalmente, o garrote era solto, e o scalp retirado cuidadosamente para se evitar o rompimento da veia e consequentes prejuízos nas próximas avaliações. $\mathrm{O}$ tempo de cada exame foi devidamente anotado para posteriormente ser 
comparado com outros estudos. As películas radiográficas foram analisadas em negatoscópio para descrição dos vasos contrastados. Utilizaram-se, para interpretação, as descrições anatômicas de Schummer et al. (1981) para bovinos e em alterações descritas por Redden (2001a) em equinos portadores de laminite. Para as análises, padronizou-se começar pela região interfalangeana proximal, na sequência a interfalangeana distal e, por último, a extremidade da falange distal. A vascularização dos dígitos dos animais nos diferentes momentos, tendo o M0 como controle, foi avaliada de forma descritiva e priorizaram-se os vasos sanguíneos principais ou regiões como as redes vasculares. Adotou-se como modelo o estudo realizado por Baldwin e Pollitt (2010) em equinos.

Concluída a identificação dos vasos sanguíneos, escolheram-se quatro veias, que foram visualizadas em todas as radiografias, em todos os animais, para proceder à mensuração da largura, tomando como base o estudo realizado por Gogoi et al. (1982). Para a mensuração das veias, as radiografias foram fotografadas, digitalizadas e compactadas em formato JPEG (Joint Photographic Experts Group) e, posteriormente, analisadas utilizando-se o software ImageJ, versão $1.36 \mathrm{~b}$, para Windows. As medidas foram tomadas em um único ponto, medialmente, nas diferentes veias. A vascularização dos dígitos dos animais nos diferentes momentos foi avaliada de forma descritiva.

O experimento seguiu um delineamento inteiramente ao acaso, em esquema de parcelas subdividas no tempo. As médias da largura das quatro veias avaliadas foram submetidas à análise de variância (ANOVA) e comparadas pelo teste t. As diferenças estatísticas foram consideradas quando o valor de probabilidade foi menor que $5 \%(\mathrm{P}<0,05)$ entre os grupos e dentro do grupo nos diferentes momentos. Para a análise dos dados, utilizou-se o programa estatístico $\mathrm{R}$ (versão 3.3.1 - 2016 - The R Foundation for Statistical Computing).

\section{RESULTADOS}

A oligofrutose induziu acidose ruminal e metabólica em todos os animais que receberam o carboidrato, independentemente da dose oferecida, confirmada pela aferição do $\mathrm{pH}$ do líquido ruminal e pela hemogasometria. $\mathrm{O}$ grupo GI obteve valor médio de $\mathrm{pH}$ ruminal de $5.47 \pm 0.83$ e de $\mathrm{pH}$ metabólico de $7.26 \pm 0.08$, em todos os momentos. No grupo GII, a média de todos os momentos foi de $5.29 \pm 0.67$ para $\mathrm{pH}$ ruminal e $7.20 \pm 0.10$ para $\mathrm{pH}$ metabólico. $\mathrm{O}$ valor mínimo aferido do $\mathrm{pH}$ ruminal foi de 3,67 e de 6,98 para $\mathrm{pH}$ metabólico. Ambos os valores foram encontrados em um animal que recebeu a maior dose.

Nenhum animal claudicou durante as 72 horas posteriores à administração da oligofrutose intrarruminal e, ainda que o quadro clínico fosse grave, a aplicação de solução de bicarbonato a 7\%, 36 horas após a aplicação da oligofrutose, evitou o óbito. Mas a desidratação foi intensa em todos os animais, comprovada pela diarreia profusa, pela alta elasticidade da pele, pela enofltamia, pelas mucosas normocoradas, secas e pela apatia.

A realização do exame venográfico dos dígitos do membro pélvico em bovinos vivos mostrou-se ser um procedimento de dificuldade de grau médio, pois há necessidade de sedação, contenção em decúbito lateral e imobilização, para impedir a movimentação. Quando a imobilização não foi perfeita, a movimentação do animal dificultou a canulação da veia em dois animais $(33,33 \%)$. Nesses, foi necessário repetir a aplicação do contraste e, para isso, respeitou-se um tempo médio de 10 minutos para a segunda aplicação.

Durante o exame, foram necessários quatro auxiliares para sistematizar as seguintes ações: manuseio do aparelho radiográfico, contenção do animal, imobilização do membro locomotor, canulação da v. digital dorsal comum III, aplicação do contraste e posicionamento do chassi. O treinamento prévio da equipe foi fundamental para a obtenção das imagens radiográficas. $\mathrm{O}$ tempo médio para a realização do exame nas duas posições radiográficas foi de $54,25 \pm 12,63$ segundos.

A quantidade de $20 \mathrm{~mL}$ de contraste foi suficiente para contrastar os vasos dos dígitos, e o procedimento ocorreu sem transtornos e de forma integral em quatro animais $(66,7 \%)$. Não foi possível canular a v. digital dorsal comum III para realizar o exame radiográfico após 36 horas 
de administração de oligofrutose em um animal $(16,7 \%)$ e aos 30 dias (M3) em outro bovino $(16,7 \%)$. Em relação à progressão do contraste, em um animal a progressão não foi completa no dígito lateral e, em outro, essa intercorrência aconteceu no dígito medial. Em ambos os animais, o insucesso foi identificado em todos os momentos.

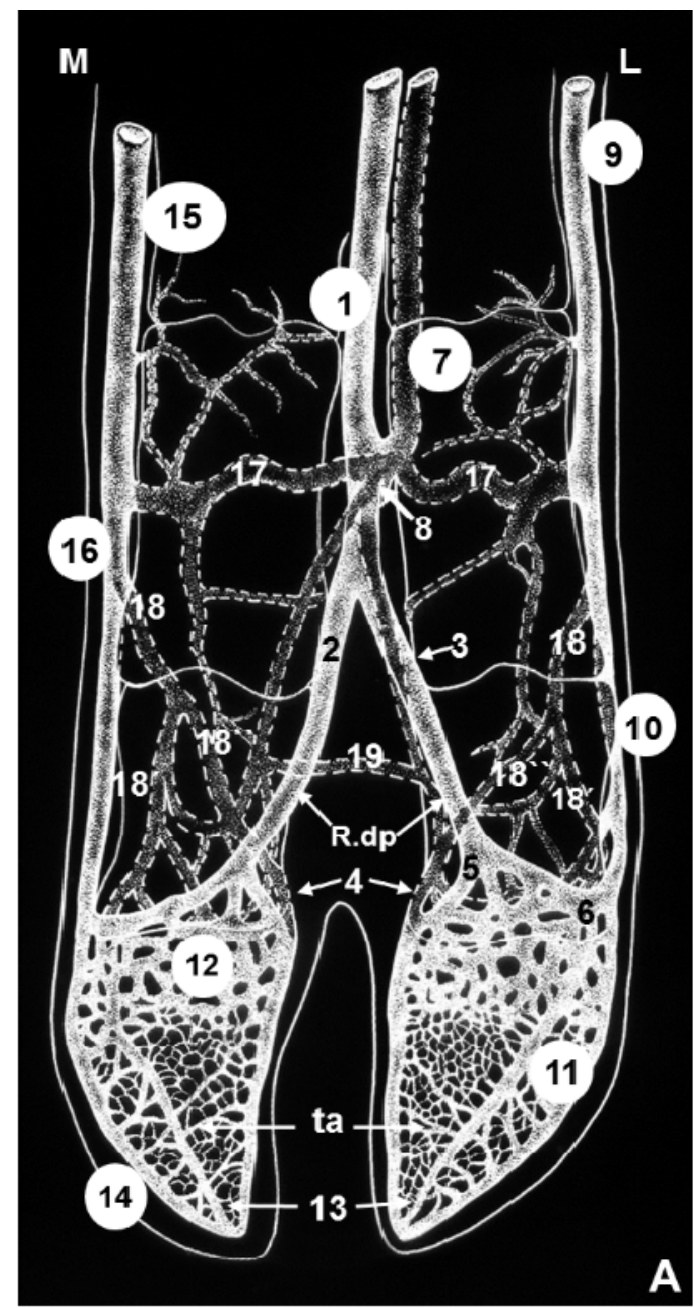

$\mathbf{A}$
Os vasos contrastados e identificados pelo exame venográfico dos dígitos de bovinos jovens, apesar de algumas diferenças individuais, permitiram a definição de um padrão anatômico. Dessa forma, foi possível descrever anatomicamente os vasos visualizados nas radiografias em posição dorsoplantar (Fig. 1) e em posição lateromedial (Fig. 2). Foram visualizadas 25 estruturas, incluindo veias, artérias e rede vascular.

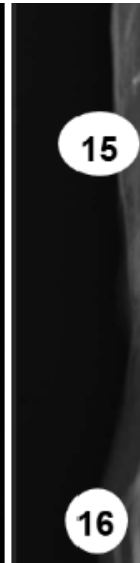

16 18 2 $18^{\prime} 18^{\prime}$

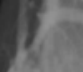

6 5

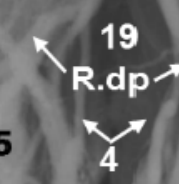

12

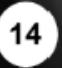

14

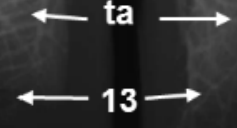

11

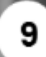

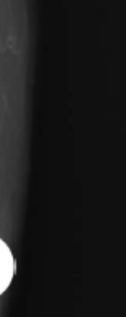

B

Figura 1. Esquema (A) e radiografia (B) dos vasos sanguíneos do pé de bovinos em visão dorsoplantar. 1) v. digital dorsal comum III; 2) v. digital dorsal própria III axial; 3) v. digital dorsal própria IV axial; 4) v. coronal profunda; 5) r. axial da v. coronal superficial; 6) r. abaxial da v. coronal superficial; 7) v. digital plantar comum III; 8) v. interdigital; 9) v. digital plantar própria IV abaxial; 10) v. do coxim digital ou v. bulbar; 11) rede venosa dorsal do cório parietal; 12) rede venosa do cório coronário; 13) v. dorsal da falange distal; 14) v. solear marginal; 15) v. digital plantar comum II; 16) v. digital plantar própria III abaxial; 17) r. plantar da falange proximal; 18) r. plantar para o coxim digital; 18') r. plantar para o coxim digital abaxial; 18') r. plantar para o coxim digital axial; 19) r. anastomótico interdigital. R.dp - r. dorsais da falange média,; ta - arco terminal; M - medial (dedo III); L - lateral (dedo IV). 

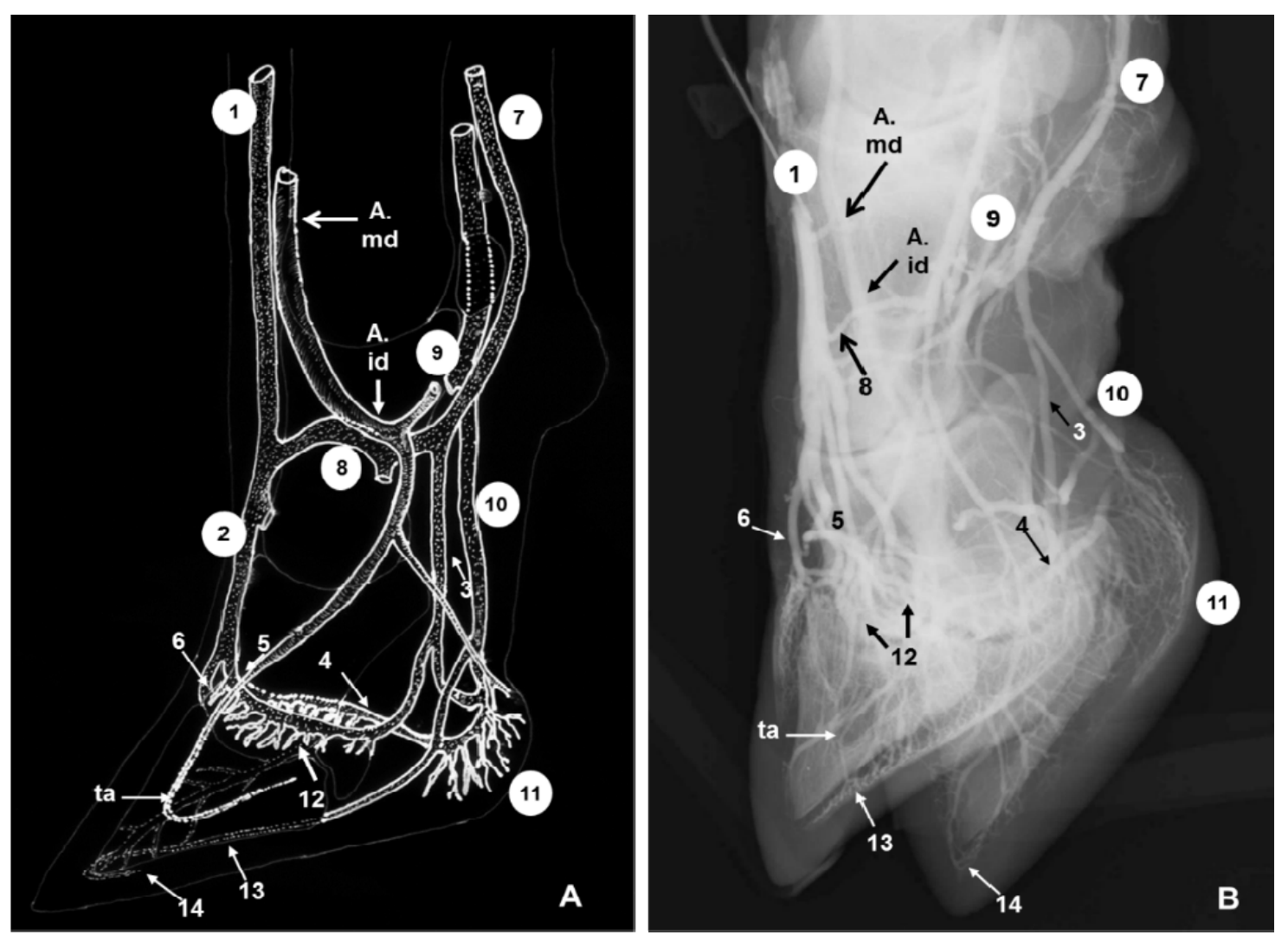

Figura 2. Esquema (A) e radiografia (B) dos vasos sanguíneos do pé de bovinos em visão dorsoplantar. 1) $\mathrm{v}$. digital dorsal comum III; 2) v. digital dorsal própria IV axial; 3) v. digital plantar própria IV axial; 4) v. coronal profunda; 5) r. axial da v. coronal superficial; 6) r. abaxial da v. coronal superficial; 7) v. digital plantar comum III; 8) v. interdigital; 9) v. digital plantar própria IV abaxial; 10) v. do coxim digital ou v. bulbar; 11) rede bulbar; 12) rede venosa do cório coronário; 13) v. solear marginal; 14) v. dorsal da falange distal. A.md - a. metatársica dorsal III; A.id - a. interdigital; ta - arco terminal.

Em dois animais (33,3\%), ocorreu constrição dos vasos com 36 horas após a aplicação da oligofrutose, e esta persistiu até a última avaliação (M3). Nos demais quatro animais $(66,7 \%)$, com 36 horas, os vasos mostraram-se mais calibrosos e em maior número, diminuindo nos momentos subsequentes (Fig. 3).

Em dois dos quatro animais citados, notou-se o desaparecimento da vascularização dos dígitos laterais no momento M3. A vascularização da rede do cório coronário e da rede bulbar também se mostrou deficiente com o passar do tempo em todos os animais (Fig. 3), sendo a alteração melhor identificada no dígito lateral. Nos animais que compuseram o GII, todas essas as alterações vasculares foram mais evidentes.
O r. plantar para o coxim digital foi mensurado em posição dorsoplantar, nos dígitos lateral e medial. As veias digital dorsal comum III e dorsal da falange distal foram avaliadas apenas em exposição lateromedial, o que permitiu somente uma mensuração. As larguras do r. plantar para o coxim digital e da v. dorsal da falange distal foram aferidas no ponto médio deles. A v. digital dorsal comum III teve a largura aferida no ponto de encontro com a $\mathrm{v}$. interdigital.

Ao se compararem os efeitos produzidos pelas dosagens $(13 \mathrm{~g} / \mathrm{kg}$ e $17 \mathrm{~g} / \mathrm{kg})$ de oligofrutose (Tab. 1), observou-se que a mensuração da largura dos vasos não sofreu $(\mathrm{P} \geq 0,05)$ interferência quando se compararam os grupos GI e GII. A exceção: a menor $(P \leq 0,05)$ largura notada foi no $r$. plantar para o coxim digital do dígito medial. 

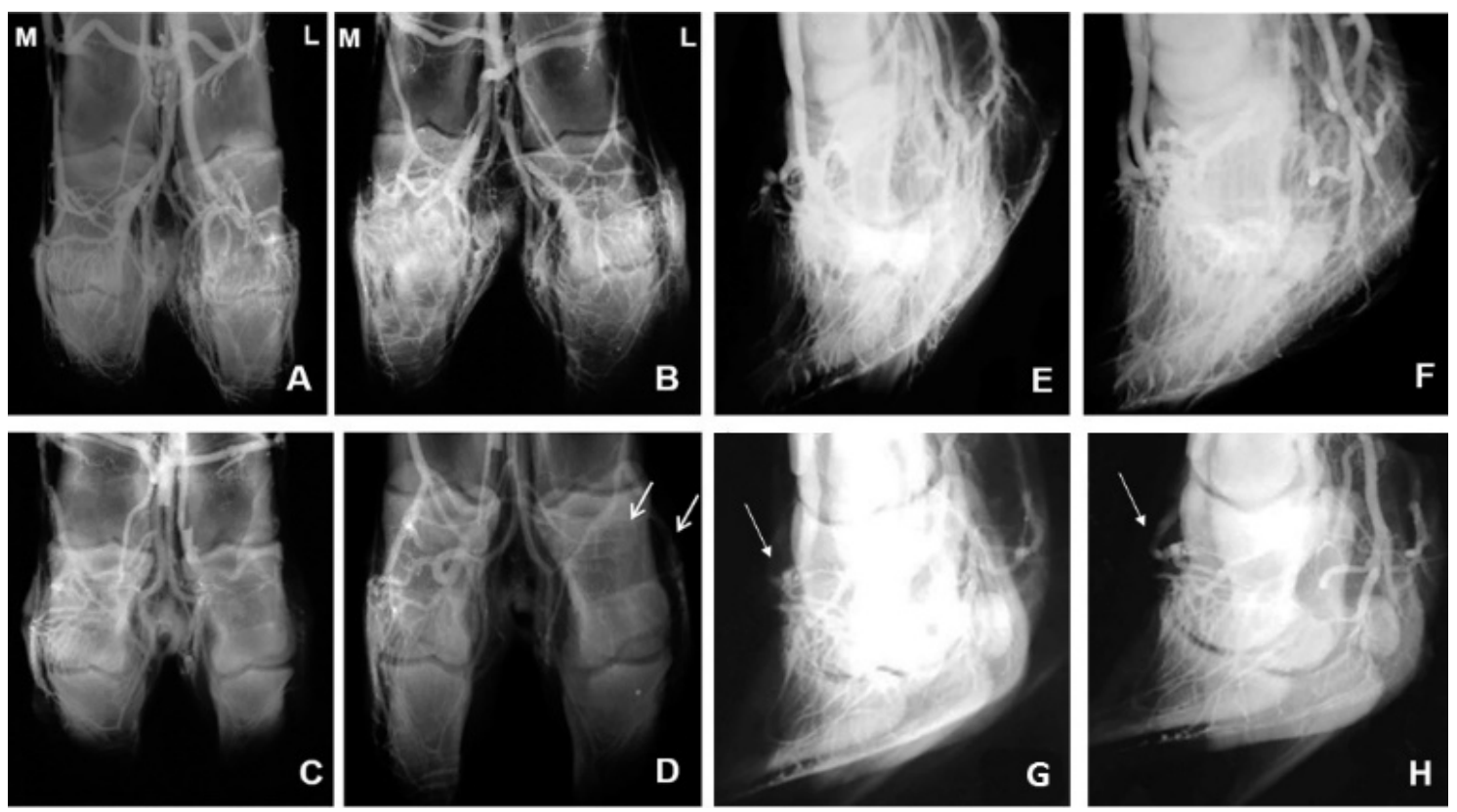

Figura 3. Imagens radiográficas dorsoplantares $(A, B, C$ e D) e lateromediais $(E, F, G$ e H) dos dígitos dos membros pélvicos de um bovino, mostrando a vascularização antes e após indução de acidose ruminal. (A) momento antes da indução com a vascularização padrão; (B) 36h após, destaque para o aumento da vascularização; (C) sete dias após, constrição de vasos principais, destaque para o desaparecimento da vascularização no dígito lateral; (D) 30 dias após, reaparecimento de alguns vasos (seta); (E) momento antes da indução, padrão controle da vascularização; (F) 36h após, aumento da vascularização; (G) sete dias e (H) 30 dias após, constrição da rede vascular com destaque para o cório coronário (setas). M dígito medial; $\mathrm{L}$ - dígito lateral.

Quando foram avaliados os vasos sanguíneos ao longo dos momentos, verificou-se que a v. digital dorsal comum III (DDCIII) mostrou-se diferente estatisticamente apenas entre M1 e M3, nos dois grupos, significando que houve uma vasodilatação inicial quando a DDCIII induziu a acidose; no final da avaliação, o valor voltou próximo ao basal (M0).

Tabela 1. Médias e desvios-padrão da largura das diferentes veias dos animais nos momentos antes (M0) da aplicação e 36 horas (M1), sete dias (M2) e 30 dias (M3) após aplicação intrarruminal de diferentes doses de oligofrutose $(\mathrm{GI}=13 \mathrm{~g} / \mathrm{kg}, \mathrm{GII}=17 \mathrm{~g} / \mathrm{kg})$

\begin{tabular}{|c|c|c|c|c|c|}
\hline \multirow{3}{*}{ Veias } & \multirow{3}{*}{ Grupos } & \multicolumn{4}{|c|}{ Momentos } \\
\hline & & M0 & M1 & M2 & M3 \\
\hline & & antes & 36 horas & sete dias & 30 dias \\
\hline \multirow{2}{*}{ DDCIII } & GI & $0.387 \pm 0.023^{\mathrm{ab}}$ & $0.479 \pm 0.103^{\mathrm{a}}$ & $0.342 \pm 0.035^{\mathrm{ab}}$ & $0.378 \pm 0.063^{b}$ \\
\hline & GII & $0.406 \pm 0.084^{\mathrm{ab}}$ & $0.445 \pm 0.085^{\mathrm{a}}$ & $0.389 \pm 0.028^{\mathrm{ab}}$ & $0.341 \pm 0.029^{b}$ \\
\hline \multirow{2}{*}{ RCDL } & GI & $0.120 \pm 0.063$ & $0.197 \pm 0.037$ & $0.105 \pm 0.071$ & $0.119 \pm 0.082$ \\
\hline & GII & $0.102 \pm 0.022$ & $0.133 \pm 0.080$ & $0.158 \pm 0.090$ & $0.144 \pm 0.144$ \\
\hline \multirow{2}{*}{ RCDM } & GI & $0.181 \pm 0.033$ & $0.204 \pm 0.031$ & $0.190 \pm 0.029$ & $0.195 \pm 0.032^{\mathrm{A}}$ \\
\hline & GII & $0.190 \pm 0.018^{\mathrm{a}}$ & $0.155 \pm 0.041^{\mathrm{ab}}$ & $0.141 \pm 0.033^{\mathrm{bc}}$ & $0.087 \pm 0.079^{\mathrm{cB}}$ \\
\hline \multirow{2}{*}{ VDFD } & GI & $0.104 \pm 0.020$ & $0.119 \pm 0.031$ & $0.101 \pm 0.071$ & $0.109 \pm 0.035$ \\
\hline & GII & $0.102 \pm 0.071$ & $0.109 \pm 0.002$ & $0.147 \pm 0.045$ & $0.104 \pm 0.013$ \\
\hline
\end{tabular}

DDCIII - v. digital dorsal comum III; RCDL - r. plantar para o coxim digital do dígito lateral; RCDM - r. plantar para o coxim digital do dígito medial; VDFD - v. dorsal da falange distal. Letras minúsculas diferentes nas linhas e letras maiúsculas diferentes nas colunas indicam diferenças estatísticas pelo teste $\mathrm{t}$ $(\mathrm{P} \leq 0,05)$. 


\section{DISCUSSÃO}

Ainda que a oligofrutose, nas doses de 13 e $17 \mathrm{~g} / \mathrm{kg}$, tenha induzido acidose ruminal em bovinos jovens, resultando em alterações significativas nos exames realizados, isso não foi suficiente para desencadear claudicação nos animais avaliados. Esse resultado pode ser consequência da administração de solução de bicarbonato, contrastando com os resultados de Thoefner et al. (2004), em que os animais, mesmo com o tratamento claudicaram. Outra possibilidade de os animais não terem claudicado seria o baixo peso dos bovinos, pois a mesma dose empregada nos animais do presente estudo desencadeou claudicação em novilhas com média de peso maior (Danscher et al., 2009). Além disso, o tipo de piso dos piquetes (gramínea) pode ter interferido, uma vez que piso de concreto favorece o desenvolvimento desse sinal clínico, com consequente laminite (Cook et al., 2004; Cook e Nordlund, 2009).

Portanto, as implicações decorrentes da indução da acidose ruminal e metabólica e, consequentemente, a claudicação, ainda precisam ser mais bem avaliadas. Para isso, a reprodução experimental da laminite em bovinos é considerada uma alternativa a ser seguida e pode contribuir substancialmente para as pesquisas envolvendo o tema. Ainda sobre o assunto, pouco é retratado sobre as alterações vasculares desde a instalação da acidose ruminal e consequente laminite, mesmo que autores tenham direcionado seus estudos para esclarecer o papel das alterações vasculares decorrentes da laminite (Sadeghi et al., 2008; Baldwin e Pollitt, 2010).

Em bovinos, a técnica venográfica foi recentemente demonstrada (Santos et al., 2016), e o tempo gasto para realização do exame é o mesmo do que se encontra em trabalhos sobre equinos (Redden, 2001a). No trabalho em questão, mesmo que o tempo médio para a realização das radiografias tenha sido um pouco acima do que foi considerado ideal para esses autores, as imagens foram consideradas de boa qualidade. Loureiro (2013), quando utilizou bovinos adultos como modelo experimental, afirmou que o tempo de até 120 segundos é compatível com imagens radiográficas de qualidade. Além da quantidade de contraste e do tempo de realização da radiografia, o $\mathrm{kVp}$, o
mAs e a distância do foco com o filme mostraram-se suficientes para visualizar estruturas de menor densidade, como a v. solear marginal.

Problemas como a falta de preenchimento vascular e o extravasamento de contraste por rompimento da veia canulada ou por perda da conexão venosa foram semelhantes aos visualizados em equinos (D'Arpe e Bernardini, 2010), mesmo que a técnica utilizada no presente estudo tenha sido adaptada. Nos casos que resultaram em perda da conexão venosa e extravasamento de contraste, a canulação foi refeita e, mesmo que ainda houvesse contraste depositado no tecido perivascular, este não foi suficiente para impedir que os vasos fossem contrastados. Portanto, o exame pode ser refeito imediatamente, o que contrasta com o tempo sugerido por Rucker et al. (2006). Mas a falta de preenchimento vascular não foi descrita em bovinos (Loureiro, 2013). Em equinos, essa falha pode estar relacionada à venocompressão temporária pelo excesso de apoio no membro durante a contenção e realização do exame (Rucker, 2010a), o que talvez não se aplique neste estudo, uma vez que não se utilizava o membro apoiado ao solo para realizar a venografia.

O mínimo de quatro auxiliares para conduzir o exame venográfico foi suficiente para sistematizar a sequência de ações e garantir que toda a metodologia fosse executada. Embora a equipe constituída tenha sido fundamental para realizar o exame, muitos autores (D'Arpe e Bernardini, 2010; Rucker, 2003/2010a/2010b) não abordaram esse aspecto em suas pesquisas. Contudo, apesar de todas as dificuldades inerentes à técnica de venografia empregando animais vivos, o exame representa um avanço no estudo da anatomia e das alterações vasculares dos dígitos dos bovinos. Neste estudo, ao tentar descrever a anatomia dos vasos dos dígitos do membro pélvico de bovinos, empregando a venografia, uma dificuldade técnica foi a sobreposição dos vasos plantares e dorsais, além do contraste simultâneo de algumas artérias. A explicação encontrada para o aparecimento de artérias nas imagens radiográficas pode ser a presença de anastomoses arteriovenosas (Danscher et al., 2011). 
Após a identificação da anatomia vascular, de forma semelhante ao que foi feito nos estudos de Santos et al. (2015), foi possível avaliar as alterações ocorridas na vascularização dos dígitos em diferentes momentos depois da manifestação clínica da acidose ruminal. Dentre as alterações, supõe-se que a vasodilatação das veias principais dos dígitos, averiguada com 36 horas após a aplicação da oligofrutose, esteja relacionada com a liberação de histamina e a endotoxemia causada pela acidose ruminal (Nocek, 1997; Hood, 1999). Como nas avaliações subsequentes, os vasos apresentaramse constritos ou ausentes. Esses achados são condizentes com os encontrados em equinos (Pollitt e Davies, 1998; Dehghani, 2008; Baldwin e Pollitt, 2010) e em bovinos (Sadeghi et al., 2008; Nurbakhsh et al., 2009) portadores de laminite. Independentemente de os animais do presente estudo não terem manifestado a claudicação, sinal presente na laminite, as alterações vasculares foram semelhantes às encontradas na afecção.

Pondera-se que a diferença estatística encontrada entre a largura da v. digital dorsal comum III nos diferentes momentos de avaliação indica que a vasodilatação inicial e o posterior retorno ao diâmetro basal coincidem com os eventos vasculares dos dígitos, apontados nos diferentes trabalhos sobre a laminite (Nurbakhsh et al., 2009; Baldwin e Pollitt, 2010). Argumenta-se ainda que a intensidade da vasoconstrição foi dose dependente, ou seja, quanto maior a dose, maior a diminuição do lúmen vascular. Entretanto, nenhum estudo fez a comparação das alterações vasculares após aplicação de diferentes doses de oligofrutose. Thoefner et al. (2004) foram os únicos a mencionar que quanto maior a dose do carboidrato de alta fermentação oferecido, mais grave são as alterações gerais decorrentes da acidose ruminal.

A diminuição da vascularização da região do cório coronário no decorrer do tempo, após a indução da acidose ruminal, foi outro achado compatível com as alterações decorrentes da laminite. Resultado semelhante foi encontrado por Baldwin e Pollitt (2010), que também utilizaram a oligofrutose para induzir experimentalmente a laminite em equinos. Por último, ao se analisarem os resultados encontrados no presente estudo e confrontá-los com os achados publicados nas pesquisas conduzidas com equinos e bovinos, fica evidente a complexidade da etiopatogenia da laminite, sendo este estudo de grande importância para a melhor compreensão dos eventos da laminite e para a padronização da anatomia vascular das extremidades distais dos membros locomotores dos bovinos.

\section{CONCLUSÃO}

O exame venográfico dos dígitos de bovinos jovens, mestiços (Bos indicus x Bos taurus), permite estudar a vascularização e identificar alterações vasculares dos dígitos dos membros pélvicos dessa espécie animal.

\section{REFERÊNCIAS}

BALDWIN, G.I.; POLLITT, C.C. Progression of venographic changes after experimentally induced laminitis. Vet. Clin. N. Am. Equine Pract., v.26, p.135-140, 2010.

COOK, N.B.; NORDLUND, K.V. The influence of the environment on dairy cow behavior, claw health and herd lameness dynamics. Vet. J., v.179, p.360-369, 2009.

COOK, N.B.; NORDLUND, K.V.; OETZEL, G.R. Environmental influences on claw horn lesions associated with laminitis and subacute ruminal acidosis in dairy cows. J. Dairy Sci., v.87, p.E36-E46, 2004.

D'ARPE, L.; BERNARDINI, D. Digital venography in horses and its clinical application in Europe. Vet. Clin. N. Am. Equine Pract., v.26, p.339-359, 2010.

DANSCHER, A.M.; ENEMARK, J.M.D.; TELEZHENKO, E. et al. Oligofructose overload induces lameness in cattle. J. Dairy Sci., v.92, p.607-616, 2009.

DANSCHER, A.M.; THOEFNER, M.B.; HEEGAARD, P.M.H. et al. Acute phase protein response during acute ruminal acidosis in cattle. Livest. Sci., v.135, p.62-69, 2011.

DEHGHANI, S.N. Digital vascular variations in normal and laminitic foot in dairy cattle. In: INTERNATIONAL SYMPOSIUM ON LAMENESS IN RUMINANTS, 15., 2008, Kuopio. Proceendings... Kuopio: [s.n], 2008. p.67-71. (Resumo). 
GOGOI, S.N.; NIGAM, J.M.; SINGH， A.P. Angiographic evaluation of bovine foot abnormalities. Vet. Radiol., v.23, p.171-174, 1982.

HOOD, D.M. The pathophysiology of developmental and acute laminitis. Vet. Clin. N. Am. Equine Pract., v.15, p.321-343, 1999

LOUREIRO, M. Estudo da técnica de venografia dos dígitos de vacas. 2013. 99f. Tese. (Doutorado em Biotecnologia Animal) Universidade Estadual Paulista, Faculdade de Medicina Veterinária e Zootecnia, Botucatu, SP.

NOCEK, J.E. Bovine acidosis: implications on laminitis. J. Dairy Sci., v.80, p.1005-1028, 1997.

NURBAKHSH, M.; NAZHVANI, S.D.; PARIZI, A.M.; TADJALLI, M. Arteriographic evaluation of laminitis digits in the hind limbs of dairy cattle. Iran. J. Vet. Surg., v.4, p.19-23, 2009.

POLLITT, C.C.; DAVIES, C.T. Equine laminitis: its development coincides with increased sublamellar blood flow. Equine Vet. J., Suppl., p.125-132, 1998.

REDDEN, R.F. A technique for performing digital venography in the standing horse. Equine Vet. Educ., v.13, p.128-134, 2001 b.

REDDEN, R.F. Possible therapeutic value of digital venography in two laminitic horses. Equine Vet. Educ., v.13, p.125-127, 2001a.

RUCKER, A. Clinical applications of digital venography. J. Equine Vet. Sci., v.30, p.491-503, $2010 b$.

RUCKER, A. Equine venography and its clinical application in North America. Vet. Clin. N. Am. Equine Pract., v.26, p.167-177, 2010a.

RUCKER, A. Interpreting venograms: normal or abnormal artifacts that may be misinterpreted. In: BLUEGRASS, LAMINITIS SYMPOSIUM, 16. 2003, Kentucky. Proceedings... Kentucky: [s.n], 2003. p.124-127. (Resumo).
RUCKER, A.; REDDEN, R.F.; ARTHUR, E.G. et al. How to perform the digital venogram. In: ANNUAL CONVENTION OF THE AMERICAN ASSOCIATION OF EQUINE PRACTITIONERS, 52., 2006, San Antonio. Proceedings... San Antonio: [s.n], 2006. p.526530. (Resumo).

SADEGHI, M.; NAZHVANI, F.; NAZHVANI, S.; SUDBAKSH, S. Digital vascular variations in normal and laminitic foot in dairy cattle. In: INTERNATIONAL SYMPOSIUM ON LAMENESS IN RUMINANTS, 15., 2008, Kuopio. Proceedings... Kuopio: [s.n], 2008. p.57-61. (Resumo).

SANTOS, I.F.C.; HUSSNI, C.A.; RODRIGUES M.J. et al. Digital venography in ruminants - a review. Vet. Q., v.26, p.22-29, 2016.

SANTOS, I.F.C.; HUSSNI, C.A.; RODRIGUES M.J. et al. Técnica venográfica constrastada in vivo dos dígitos de ovinos e caprinos. Arq. Bras. Med. Vet. Zootec., v.67, p.1630-1638, 2015.

SCHUMMER, A.; WILKENS, H.; VOLLMERHAUS, B.; HABERMEHL, K. The anatomy of the domestic animals. The circulatory system, the skin, and the cutaneous organs of the domestic mammal. 3.ed. Berlin: Verlag Paul Parey, 1981. 557p.

SPRECHER, D.J.; HOSTETLER, D.E.; KANEENE, J.B. A lameness scoring system that uses posture and gait to predict dairy cattle reproductive performance. Theriogenology, v.47, p.1179-1187, 1997.

THOEFNER, M.; POLLITT, C.; VAN EPS, A. et al. Acute bovine laminitis: a new induction model using alimentary oligofructose overload. J. Dairy Sci., v.87, p.2932-2940, 2004.

THOEFNER, M.; WATTLE, O.; POLLITT, C. et al. Histopathology of oligofructose-induced acute laminitis in heifers. J. Dairy Sci., v.88, p.2774-2782, 2005. 